\title{
Advances in cancer early diagnosis with liquid biopsy-based approaches
}

\author{
Wei Zhang ${ }^{1,2}$ \\ 'Department of Preventive Medicine, Northwestern University Feinberg School of Medicine, Chicago, IL 60611, USA. \\ ${ }^{2}$ The Robert H. Lurie Comprehensive Cancer Center, Northwestern University Feinberg School of Medicine, Chicago, IL 60611, \\ USA.
}

Correspondence to: Dr. Wei Zhang, Department of Preventive Medicine, Northwestern University Feinberg School of Medicine, 680 N. Lake Shore Dr., Suite 1400, Chicago, IL 60611, USA. E-mail: wei.zhang1@northwestern.edu

How to cite this article: Zhang W. Advances in cancer early diagnosis with liquid biopsy-based approaches. J Cancer Metastasis Treat 2021;7:22. https://dx.doi.org/10.20517/2394-4722.2021.82

Received: 31 Mar 2021 Accepted: 7 Apr 2021 Published: 23 Apr 2021

Academic Editor: Lucio Miele Copy Editor: Xi-Jun Chen Production Editor: Xi-Jun Chen

Significant progress in cancer diagnosis and treatment has contributed to improved clinical outcomes for many cancers. However, cancer remains a major world-wide health problem with an estimated 19.3 million new cases and almost 10.0 million cancer deaths in $2020^{[1]}$. Early detection of malignant tumors that are still treatable (e.g., surgical removal) is key to lowering cancer-related mortality and improving patient survival. Compared to tissue-based cancer diagnosis approaches that may be limited by the issue of tumor heterogeneity, accessibility to a tumor, and possible complications associated with the biopsy procedure, approaches targeting liquid biopsies such as blood, urine, cerebrospinal fluid, saliva, and sweat have been demonstrated for their clinical potential as a noninvasive or minimally-invasive alternative. Specifically, tumor-derived mutational, epigenetic, and transcriptomic features are contained in liquid biopsies collected from a patient, providing the basis for developing effective diagnostic biomarkers from these specimens. Of note, during the past few years, exciting technical advances have emerged to allow profiling various molecular targets in a variety of clinical specimens. In this special issue of "Liquid Biopsies in Cancer Early Diagnosis", six reviews and original articles showcased the current advances in this fast-developing field of research.

The four reviews in this special issue provided timely summaries of related research from a perspective of an individual cancer or cancers in general. D'Amico et al. ${ }^{[2]}$ reviewed clinical evidence for the utility of liquid biopsy in detecting early breast cancer. They argued that combining molecular information from liquid 
biopsy or integration with sensitive and complementary ctDNA technologies may be the key for the practice-changing implementation of liquid biopsy in managing early breast cancer. Endometrial cancer, another women's cancer, is the focus of a review by Malentacchi et al. ${ }^{[3]}$. Endometrial cancer is usually detected at low stage with a good prognosis, though some cases can evolve to bad prognosis. However, there is no molecular target for treatment, detection, or monitoring. Through a comprehensive review of molecular targets in liquid biopsies, Malentacchi et al. ${ }^{[3]}$ discussed how these approaches may help in the management and characterization of patients affected by endometrial cancer. Cancer-derived exosomes carry a unique set of DNA, RNA, protein and lipid that may serve as diagnostic biomarkers. Specifically, Happel et al. ${ }^{[4]}$ reviewed scientific highlights in biomarker discovery of exosome-based extracellular RNA (exRNA) in human cancers. Their review suggested that exRNA is useful not only for liquid biopsies to diagnose various cancer types, but it also provides potential avenues for therapy. Numerous new technologies are being developed for liquid biopsy-based diagnosis. Su et al. ${ }^{[5]}$ were particularly interested in magnetic nanotechnologies that possess many advantages such as low background noise, high sensitivity, short assay time, and the ability to detect multiple biomarkers at the same time. Specifically, Su et al..$^{[5]}$ focused their discussion on the recent advances of magnetic nanotechnologies in liquid biopsies for sensitive, efficient, and portable cancer diagnosis.

Both of the original articles focused on lung cancer. Rath et al ${ }^{[6]}$ investigated a new mechanism of drug resistance for small-cell lung cancer (SCLC) circulating tumor cells (CTCs). They measured cellular fragments in SCLC CTC cell lines and compared to permanent SCLC lines. Their findings suggested that generation of cell-associated debris by SCLC CTCs may offer protective effects against cytotoxic drugs, representing a novel mechanism allowing survival of SCLC CTCs in patients. In comparison, Udukala et al. ${ }^{[7]}$ described their pilot study of early detection of non-small cell lung cancer (NSCLC) in liquid biopsies by ultrasensitive protease activity analysis. Specifically, using a case-control design, they presented promising results for the feasibility of minimally-invasive early diagnosis of NSCLC by protease biomarkers in the sera derived from peripheral blood.

During the past decade, the research community has achieved tremendous advances in cancer early diagnosis with liquid biopsy-based approaches. Future investigations will ultimately prove the utility of these novel approaches in improving clinical outcomes of cancer patients.

\section{DECLARATIONS}

\section{Authors' contributions}

The author contributed solely to the article.

\section{Availability of data and materials}

Not applicable.

\section{Financial support and sponsorship}

This work was partially supported by grants from the National Institutes of Health: R01CA223662, R21MD011439, and U01CA217078.

\section{Conflicts of interest}

The author is a shareholder of Shanghai Epican Genetech Co. Ltd, which develops liquid biopsy-based cancer biomarkers. This article is not related to the company. 


\section{Ethical approval and consent to participate}

Not applicable.

\section{Consent for publication}

Not applicable.

\section{Copyright}

(c) The Author(s) 2021.

\section{REFERENCES}

1. Sung H, Ferlay J, Siegel RL, et al. Global cancer statistics 2020: GLOBOCAN estimates of incidence and mortality worldwide for 36 cancers in 185 countries. CA Cancer J Clin 2021. DOI PubMed

2. D'Amico P, Corvaja C, Gerratana L, et al. The use of liquid biopsy in early breast cancer: clinical evidence and future perspectives. $J$ Cancer Metastasis Treat 2021;7:3. DOI

3. Malentacchi F, Sgromo C, Antonuzzo L, et al. Liquid biopsy in endometrial cancer. J Cancer Metastasis Treat 2020;6:34. DOI

4. Happel C, Ganguly A, Tagle DA. Extracellular RNAs as potential biomarkers for cancer. J Cancer Metastasis Treat 2020;6:32. DOI

5. Su D, Wu K, Saha R, et al. Magnetic nanotechnologies for early cancer diagnostics with liquid biopsies: a review. J Cancer Metastasis Treat 2020;6:19. DOI

6. Rath B, Plangger A, Moser D. et al. Protection of small-cell lung cancer circulating tumor cells by cellular fragmentation. $J$ Cancer Metastasis Treat 2020;6:30. DOI

7. Udukala DN, Wendel SO, Wang H. et al. Early detection of non-small cell lung cancer in liquid biopsies by ultrasensitive protease activity analysis. $J$ Cancer Metastasis Treat 2020;6:25. DOI 